\title{
The declination degeneracy in interferometric astrometry
}

\author{
D. Buscher
}

\author{
Cavendish Laboratory, JJ Thomson Avenue, Cambridge CB3 0HE, UK \\ e-mail: dfb@mrao.cam.ac.uk
}

Received 3 November 2011 / Accepted 14 January 2012

\begin{abstract}
Context. Interferometric astrometry at radio and optical wavelengths serves as a fundamental and high-precision method for measuring the positions of celestial objects and for geodetic and Earth orientation measurements.

Aims. We determine the importance and form of the degeneracy which arises when the interferometric baselines and the celestial target positions are estimated simultaneously from the same data under circumstances where the declination range of the targets is limited.

Methods. Heuristic analyses of an idealised interferometric experiment were used to determine how a degeneracy could arise as a result of limited declination coverage and what form it might take. Singular Value Decomposition of simulated experiments with different declination coverages and different hour-angle coverages were then used to quantify the level of degeneracy present and the form of the degeneracy in example cases.

Results. A significant degeneracy was found which depends strongly on the declination coverage and less strongly on the hour-angle coverage of the experiment. The degeneracy is significant even when the declination coverage extends from the pole to the equator. The degeneracy takes the form of a systematic distortion of the measured coordinates in the declination direction which is larger near the equator than at the poles, together with a corresponding baseline distortion.

Conclusions. Taking into account this degeneracy in a quantitative way can have a significant effect on the design and interpretation of astrometric and geodetic experiments.
\end{abstract}

Key words. astrometry - techniques: interferometric

\section{Introduction}

Interferometric measurements allow high-sensitivity determinations of the angular positions of astronomical sources. At radio wavelengths, Very Long Baseline Interferometry (VLBI) measurements form a basis for the International Celestial Reference Frame (ICRF) (Ma et al. 1998), while at optical wavelengths there are projects pursuing astrometric interferometry either for fundamental wide-angle astrometry (Hummel et al. 1994) or for exoplanet detection using narrow-angle astrometry (Shao \& Colavita 1992; Launhardt et al. 2007).

The interferometric measurement of angular position depends on accurate knowledge of the interferometric baseline, i.e. the vector between the two telescopes or antennae whose signals are being interfered. In order to measure celestial positions at the milliarcsecond level, knowledge of the baseline to an accuracy of a few parts in $10^{9}$ is required. This kind of accuracy is difficult to obtain by direct measurement of the telescope positions, and it is standard practice to determine both the baseline and the source positions simultaneously from interferometric observations of a series of celestial sources. A useful bonus of this approach is that it yields baseline measurements that can be used to determine geodetic and Earth orientation parameters (Sovers et al. 1998).

A danger of determining baselines and source positions from the same data is that it can lead to degeneracies in the model being fit to the data, that is to say that errors in one set of model parameters can mask errors in another set. The most well-known of these degeneracies is that an error in the right ascension (RA) of all the celestial sources can be offset by an equal error in the assumed orientation of the baseline. This degeneracy is taken into account by using the interferometric measurements to determine only the relative RAs of the sources rather than determining absolute values.

Less often considered is a corresponding global degeneracy in declination. As will be demonstrated later in this paper, this degeneracy arises purely from the geometry of interferometric measurements and is present to a greater or lesser extent in any experiment which attempts to measure the baselines and source positions simultaneously. The degeneracy is in most cases only a partial one and depends both on the range of declinations of the sources which are observed and the baseline geometry employed. There is some evidence that the effects of this degeneracy are known in the literature: Thompson et al. (2001) state that to do reliable astrometry with an interferometer, one should observe sources over "as wide a declination range as possible". However, questions as to how wide this range needs to be and what the consequences of not having a wide enough range are have not to the author's knowledge been answered quantitatively.

In this paper a quantitative analysis of this degeneracy is presented. A general measurement model is developed and then a simplified scenario is used to illustrate the nature of the degeneracy in a heuristic fashion. The degeneracy is then quantified numerically for representative experimental geometries. The implications for a sample of astrometric and geodetic experiments are then discussed. 


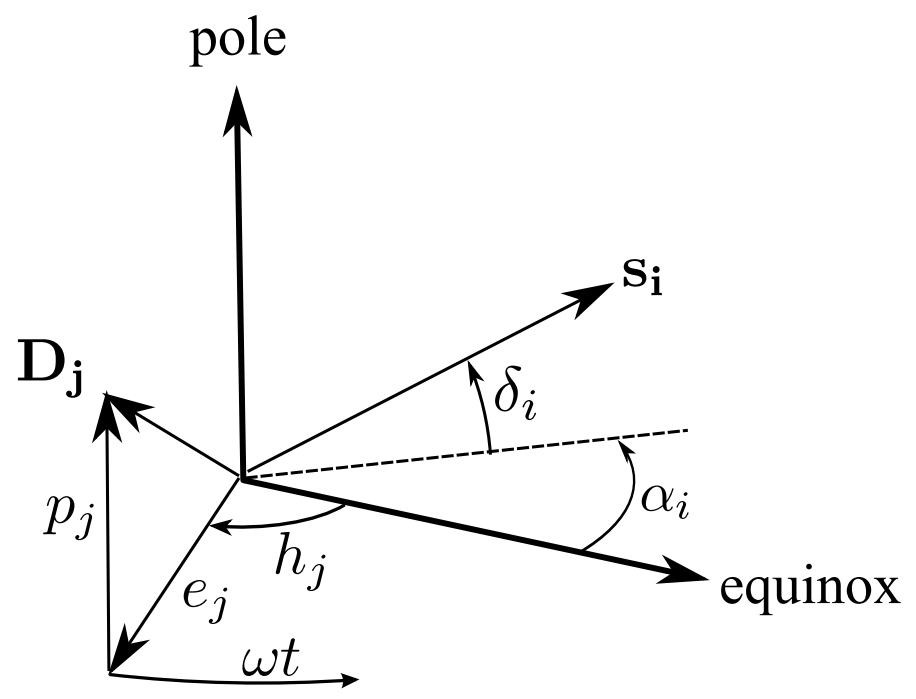

Fig. 1. Geometry for the interferometric experiment. The vector $s_{i}$ is pointing towards the a celestial object with right ascension $\alpha_{i}$ and declination $\delta_{i}$. The baseline vector $\boldsymbol{D}_{j}$ has polar and equatorial components $p_{j}$ and $e_{j}$ as shown, and makes an angle with the equinox of $h_{j}$ at time $t=0$. The baseline is rotating at angular velocity $\omega$ with respect to the celestial frame.

\section{Measurement model}

The simplified model for an interferometric astrometric experiment used here is intended to apply to ground-based interferometers in both the optical and radio wavebands, but could also be applied to a space-based interferometer on a rotating platform. The modelled interferometer consists of a set of point-like collecting elements (hereafter "telescopes" or "antennae") and it observes a set of fixed point-like celestial targets (hereafter "stars" or "sources"). The interferometer measures the difference in time of arrival ("delay") of signals arriving at different telescopes from a given star. A delay can be measured between any two telescopes in the interferometer, and depends on the instantaneous vector separation or "baseline" between the telescopes.

The delay measured for a given star and telescope pair is sampled at discrete times and the dataset consists of a series of delay measurements for each star-baseline combination, with no assumptions being made about the completeness of this set in terms of which star-baseline combinations are measured. An alternative formalism for interferometric astrometry uses the telescope coordinates rather than the baselines as the parameters to be solved for. When a given telescope is a member of more than one baseline, the telescope-based formalism may have superior noise-propagation performance compared to the baseline-based formalism used here, but we show in the Appendix that the general characteristics of the degeneracies which arise are not affected by whether or not a baseline or telescope-based formalism is used.

Figure 1 shows the coordinate system used to define the star and telescope coordinates. The telescopes are fixed in a frame which is rotating at angular velocity $\omega$ with respect to the celestial frame. The rotation axis of the frame will be referred to as the "polar" axis and the plane perpendicular to this axis as the "equatorial" plane. A particular direction in the equatorial plane which is fixed with respect to the celestial frame is denoted as the "equinox".

The stellar directions $\left\{s_{i}, i=1 \ldots N_{\text {star }}\right\}$ are measured relative to the equatorial plane and the equinox in terms of their right ascensions $\alpha_{i}$ and declinations $\delta_{i}$ as shown in the figure. It should be noted that this definition of the celestial coordinates with respect to the rotation axis at the epoch of observation means that Earth orientation effects such as precession and nutation can be ignored. The baselines $\left\{\boldsymbol{D}_{j}, j=1 \ldots N_{\text {bas }}\right\}$ are denoted in terms of the lengths of their polar and equatorial components $p_{j}$ and $e_{j}$ respectively, and the hour angle $h_{j}$ between the baseline and the equinox at time $t=0$. In radio VLBI it is conventional to denote the hour-angle separation between the source and the baseline at time $t$ in terms of the "interferometer hour angle" (IHA) given by

$H_{i j}=\omega t-h_{j}-\alpha_{i}-\pi / 2$

(Pearson 1991).

For simplicity, relativistic corrections, the effects of refraction, geophysical corrections such as those due to Earth tides, and effects due to mechanical imperfections in the telescopes are not included in the model. Given these assumptions, the $k$ th sample of the delay measured on star $i$ with baseline $j$ is given by

$d_{i j k}=p_{j} \sin \delta_{i}-e_{j} \cos \delta_{i} \sin H_{i j k}+c_{j}+n_{i j k}$,

where $H_{i j k}$ is the IHA at the time at which the delay is sampled, $n_{i j k}$ is a random "noise" error in the delay measurement and $c_{j}$ is a baseline-dependent systematic error assumed to be fixed for the duration of the experiment. This systematic error is typically due to quasi-static signal propagation delays within the interferometer and is known as the "clock offset" in radio VLBI or "constant term" in optical interferometry. This term can be considered as part of the baseline model. Note that throughout this paper "delays" are denoted in terms of an optical path difference, i.e. a distance: the corresponding time delays can be derived by dividing by the speed of light.

A change of notation is now introduced in order to linearise the problem. Let the star and baseline coordinates be written as $\left\{\alpha_{i}+\Delta \alpha_{i}, \delta_{i}+\Delta \delta_{i}\right\}$ and $\left\{e_{j}+\Delta e_{j}, p_{j}+\Delta p_{j}, h_{j}+\Delta h_{j}, c_{j}+\Delta c_{j}\right\}$ respectively where $\alpha_{i}, \delta_{i}, e_{j}, p_{j}, h_{j}$, and $c_{j}$ are a priori estimates of the star positions and baseline parameters and $\Delta \alpha_{i}, \Delta \delta_{i}, \Delta e_{j}$, $\Delta p_{j}, \Delta h_{j}$, and $\Delta c_{j}$ are unknown (but assumed small) offsets between the a priori estimates and the true values. A first-order expansion of Eq. (2) gives the difference between the measured delay and that expected from the a priori coordinates as

$$
\begin{aligned}
\Delta d_{i j k}= & \Delta p_{j} \sin \delta_{i} \\
& +\Delta \delta_{i}\left(p_{j} \cos \delta_{i}+e_{j} \sin \delta_{i} \sin H_{i j k}\right) \\
& -\Delta e_{j} \cos \delta_{i} \sin H_{i j k} \\
& +\left(\Delta \alpha_{i}+\Delta h_{j}\right) e_{j} \cos \delta_{i} \cos H_{i j k} \\
& +\Delta c_{j}+n_{i j k} .
\end{aligned}
$$

This equation represents the set of simultaneous linear equations which are to be solved for the offsets in the baselines and star positions, given the set of differences between the expected and measured delays.

\section{Heuristic exploration}

\subsection{Simplified model}

The structure of Eq. (3) can be made more clear by writing it as

$\Delta d_{i j k}=A_{i j} \cos H_{i j k}+B_{i j} \sin H_{i j k}+C_{i j}+n_{i j k}$

where

$$
\begin{aligned}
& A_{i j}=e_{j} \cos \delta_{i}\left(\Delta \alpha_{i}+\Delta h_{j}\right) \\
& B_{i j}=e_{j} \sin \delta_{i} \Delta \delta_{i}-\cos \delta_{i} \Delta e_{j} \\
& C_{i j}=\sin \delta_{i} \Delta p_{j}+p_{j} \cos \delta_{i} \Delta \delta_{i}+\Delta c_{j} .
\end{aligned}
$$


It can be seen that $A_{i j}$ and $B_{i j}$ are quadrature coefficients of a sinusoidally oscillating ("diurnal") delay and $C_{i j}$ represents the "DC" or time-invariant delay for a given combination of star and baseline.

A considerable simplification of the mathematics results from assuming that the interferometric delays can be sampled uniformly in hour angle (HA) for all the stars for a complete revolution of the rotating frame, i.e. over a complete $24-\mathrm{h}$ period for Earth-based observations. While this cannot usually be achieved in practice, the assumption will reveal the degeneracies for a "best case" set of observations.

If every star is observed at $N$ equally-spaced times within a revolution for each telescope pair then, for $N>2 \mathrm{Eq}$. (4) can be solved for the values of $A_{i j}, B_{i j}$ and $C_{i j}$ using a discrete Fourier transform, yielding three estimators $\hat{A}_{i j}, \hat{B}_{i j}$ and $\hat{C}_{i j}$ given by

$$
\begin{aligned}
& \hat{A}_{i j}=\frac{2}{N} \sum_{k=1}^{N} \cos H_{i j k} \Delta d_{i j k} \\
& \hat{B}_{i j}=\frac{2}{N} \sum_{k=1}^{N} \sin H_{i j k} \Delta d_{i j k} \\
& \hat{C}_{i j}=\frac{1}{N} \sum_{k=1}^{N} \Delta d_{i j k} .
\end{aligned}
$$

If the noise on the delay measurements is uncorrelated and has uniform variance, i.e. $\left\langle n_{i j k} n_{l m n}\right\rangle=\delta_{i l} \delta_{j m} \delta_{k n} \sigma^{2}$ where $\delta_{p q}$ is the Kronecker delta, then the orthogonality of the Fourier basis functions can be used to show that these estimators represent least squares solutions and that the estimators have uncorrelated errors of $\sigma \sqrt{\frac{2}{N}}, \sigma \sqrt{\frac{2}{N}}$ and $\sigma \sqrt{\frac{1}{N}}$ respectively. A least-squares solution to Eq. (3) then becomes equivalent to a weighted-leastsquares solution to the set of simultaneous equations

$$
\begin{aligned}
e_{j} \cos \delta_{i}\left(\Delta \alpha_{i}+\Delta h_{j}\right) & =\hat{A}_{i j} \\
e_{j} \sin \delta_{i} \Delta \delta_{i}-\cos \delta_{i} \Delta e_{j} & =\hat{B}_{i j} \\
p_{j} \cos \delta_{i} \Delta \delta_{i}+\sin \delta_{i} \Delta p_{j}+\Delta c_{j} & =\hat{C}_{i j}
\end{aligned}
$$

with $\left\{\Delta \alpha_{i}, \Delta \delta_{i}, \Delta e_{j}, \Delta p_{j}, \Delta h_{j}, \Delta c_{j}\right\}$ as unknown model parameters and $\left\{\hat{A}_{i j}, \hat{B}_{i j}, \hat{C}_{i j}\right\}$ as the data corrupted by random noise.

\subsection{Analytical degeneracies}

Any non-trivial solution to Eqs. (11) to (13) with the right-hand sides of the equations all zero represents a degeneracy in the equations, as this solution can be added to any other solution and not change the degree of fit to the data. It is clear by inspection of Eq. (11) that the solution is degenerate in $\Delta \alpha_{i}$ and $\Delta h_{j}$ because there there would be no change in the measured delays if all the source RAs were changed by some arbitrary amount and the baselines were rotated by the same amount. This is the familiar indeterminacy of the origin of right ascension for interferometric experiments.

In addition to this degeneracy in RA, there is also a degeneracy in declination. Inspection of Eq. (12) shows that if the equatorial components of the baselines are offset by

$$
\Delta e_{j}=\beta e_{j}
$$

and the declinations are simultaneously offset by

$\Delta \delta_{i}=\beta \cot \delta_{i}$ where $\beta$ is an arbitrary constant, then there is no change in the value of $\hat{B}_{i j}$, in other words we cannot distinguish between the true solution and the true solution plus a set of offsets of this form.

This degeneracy is unconstrained when the family of equations represented by Eq. (12) is taken by itself, but including the family of Eqs. (13) provides constraints. Substituting the declination distortion in Eq. (15) into (13) gives

$$
\begin{aligned}
\hat{C}_{i j} & =\Delta c_{j}+\Delta p_{j} \sin \delta_{i}+\beta p_{j} \cos ^{2} \delta_{i} / \sin \delta_{i} \\
& =\Delta c_{j}+\sin \delta_{i}\left[\Delta p_{j}-\beta p_{j}\right]+\beta p_{j} / \sin \delta_{i} \\
& =\Delta c_{j}+p_{j}\left[\left(\gamma_{j}-\beta\right) \sin \delta_{i}+\beta / \sin \delta_{i}\right]
\end{aligned}
$$

where $\gamma_{j} \equiv \Delta p_{j} / p_{j}$. This has no general solution for zero $\hat{C}_{i j}$ but non-zero $\beta$ and so when the polar component of at least one baseline is non-zero, the degeneracy is broken.

However, it is still possible to recover a partial degeneracy. If the delays are measured with some finite accuracy then there exist approximate solutions to the measurement equations which are indistinguishable to within the noise from the correct solutions. For example, setting $c_{j}=-2 \beta p_{j}$ for some constant $\beta$ and $\gamma_{j}=2 \beta$ we have

$$
\begin{aligned}
\hat{C}_{i j} & =\beta p_{j}\left(\sin \delta_{i}+1 / \sin \delta_{i}-2\right) \\
& \approx \beta p_{j} \phi_{i}^{4} / 4
\end{aligned}
$$

for small $\phi_{i}$, where $\phi_{i}=\pi / 2-\delta_{i}$. Thus for stars which are close to the pole, the change in the fit to the data is small, even for substantial distortions of the declinations. For example, if $e_{j} \approx p_{j}$ and $\hat{C}_{i j}$ is measured to an accuracy of $\sigma=\epsilon p_{j}$, one might intuitively expect to be able to measure the declination to an accuracy of order $\epsilon$ radians. For an observation of a set of sources within $30^{\circ}$ of the pole, the declinations can potentially be in error by more than 27 times this amount while changing the fit to the data by less less than $\sigma$.

For sources near the equator a distortion proportional to $\cot \delta$ would produce large misfits to the delay data, but even here it may still be possible to achieve and acceptable fit to the data by adjusting the declination offsets away from the $\cot \delta$ functional form to give a slight mismatch to the observed diurnal delay variations but less mismatch to the DC term.

\section{Quantifying the partial degeneracy}

\subsection{Singular value decomposition}

The above heuristic analysis suggests that it is possible to get a badly-conditioned system of equations if the declination range of the observed targets is not sufficiently large. To give more detailed insight into this degeneracy, the problem was studied numerically using Singular Value Decomposition (SVD). SVD is explained in detail in texts on numerical analysis (see for example Press et al. 1992; Golub \& Reinsch 1970) as a method of analyzing a set of singular (i.e. degenerate) or nearly-singular equations. Here we summarise the key details relevant to the analysis performed in this paper.

A set of simultaneous linear equations such as that defined by Eq. (3) can be written in matrix form as

$b=A x+\eta$

where $x$ is a vector whose elements are the unknown model parameters to be estimated (baselines and source positions), $A$ is the "design matrix" for the system, $b$ is a vector of data values 
(delays) and $\eta$ is a vector of random noise values. SVD decomposes the design matrix into the product of three matrices:

$A=U w V^{T}$

where $w$ is a diagonal matrix and $U$ and $V$ are unitary. The ondiagonal elements of the matrix $w$ are known as the "singular values" and are equivalent to the eigenvalues of a square matrix but generalized to the case of non-square matrix.

SVD can be used to invert the above set of equations to yield a least-squares solution for the values of $x$ given the measured values of $b$

$\hat{x}=A^{\dagger} b$

where

$A^{\dagger}=V w^{-1} U^{T}$

is the "generalised inverse" of $A$.

Importantly for this investigation, SVD yields quantitative information on any degeneracies in the matrix inversion. Consider a vector space whose axes correspond to the elements of the model parameter vector $x$, i.e. the "model space" or the "solution space". In this space, surfaces of constant $\chi^{2}$ misfit to the data (i.e. surfaces of constant likelihood if the data have Gaussian errors) have the form of an ellipsoid. The magnitudes of the principal axes of this "error ellipsoid" are proportional to the inverses of the singular values and the "directions" of the axes are given by the corresponding columns of $V$ - these columns can be considered to be "eigenvectors" of the design matrix.

In the case of a singular set of equations, one or more of these singular values is zero, and thus the error ellipsoid is infinitely extended in one or more directions. These directions correspond to the "null space" of the system of measurement, i.e. the set of linear combinations of model variables which can be altered at will without altering the measurements. For the RA degeneracy, the null space consists of a single eigenvector which shifts the right ascensions of all the stars and the hour angles of all the baselines by the same amount. For the declination degeneracy in the case where the polar components of the baselines are all zero (e.g. a set of East-West baselines) then the null space has an additional eigenvector consisting of the set of declination offsets proportional to $\cot \delta_{i}$ together with the corresponding baseline distortion.

In the case of a non-singular but partially-degenerate set of equations, like those under discussion here, the corresponding singular values will not be identically zero, but will be small in comparison to the other singular values. The corresponding linear combination of solution variables will not be completely undetermined, but will have an uncertainty which is much higher than any other linearly-independent set of variables. The error ellipsoid is highly elongated along the directions corresponding to the small eigenvalues. For the purpose of this analysis the nomenclature of singular equations is carried over and the underdetermined directions will hereafter be called the "null space", with the remaining directions being termed the "range space" of the design matrix.

\subsection{Numerical simulations}

The effects of varying the declination distribution of the sources $\left\{\delta_{i}\right\}$ was investigated using SVD. For simplicity, the initial study considered the idealised scenario where continuous observations over a complete revolution of the rotating frame are possible.

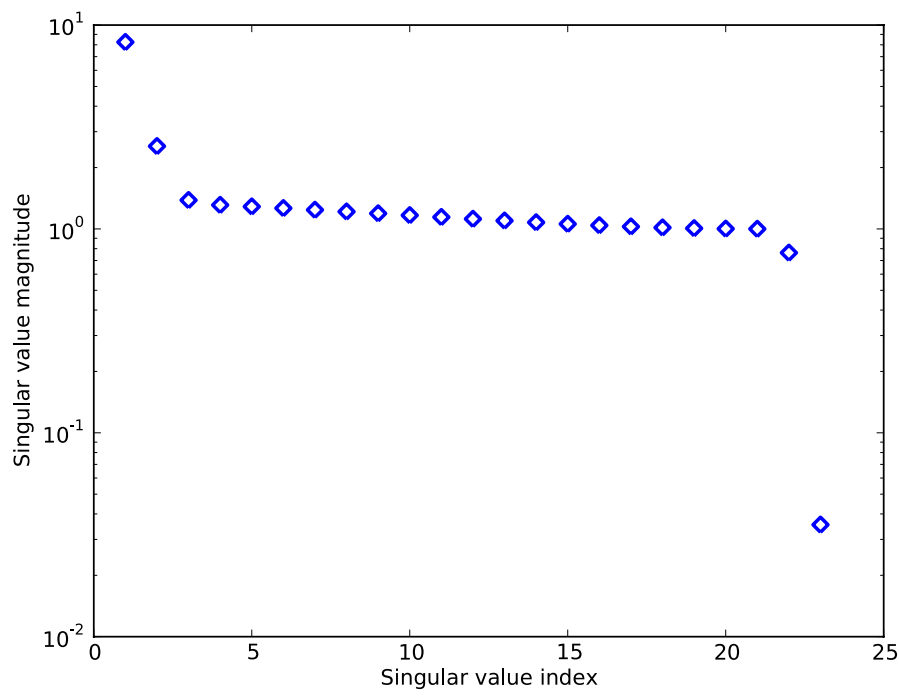

Fig. 2. Singular values for a simulated interferometric experiment. The singular values are arranged in descending order. The baseline had equal polar and equatorial components. Twenty stars equally-spaced in declination between $+30^{\circ}$ and $+90^{\circ}$ were observed, and the delay was sampled uniformly over the full $360^{\circ}$ hour angle range. One of the 23 singular values is significantly smaller than the rest, corresponding to a poorly constrained "direction" in solution space.

The equation independent of the declination offsets (Eq. (11)) was ignored and the design matrix for the system of equations represented by Eqs. (12) and (13) was formed. The factor of 2 difference between the variances of $\hat{B}_{i j}$ and $\hat{C}_{i j}$ was taken into account by appropriately scaling the rows of the matrix.

Observations of a set of stars spaced equally in declination were simulated. Typically around 20 stars were used. The declination range of the stars was varied and the singular value matrices were calculated numerically. These matrices were then used to infer the level and form of the degeneracy in declination.

It was found that the results were not affected in a qualitative manner by the number or geometry of baselines, so long as the maximum polar and equatorial components of the set of baselines were roughly comparable. This can be understood from realising that in Eqs. (12) and (13) the polar and equatorial components of any given baseline are only connected through the observation of common stars, so the polar component of one baseline can be equally important to constraining the equatorial component of another baseline as it is to constraining the equatorial component of its own baseline. For the results presented in this subsection a single baseline was used, and the equatorial and polar components of this baseline were equal in length.

Singular value decomposition confirmed that the system of equations is ill-conditioned, especially for experiments with small declination ranges. Figure 2 shows the singular values for a typical case. Most of the singular values are of similar magnitude, except for one singular value which is considerably smaller, indicating a partial degeneracy.

Degeneracies are often quantified using the "condition number" - the ratio of the maximum singular value to the minimum singular value. The condition number provides a measure of the worst-case error that could result from the solution of a set of equations, regardless of how improbable the worst-case scenario is.

An alternative figure-of-merit is used here which provides a measure of the typical rather than the worst-case degradation of 
performance due to the presence of a degeneracy. It compares the contribution to the total variance from the degenerate axis of the error ellipsoid to the average contribution from the remaining axes.

The total variance of the least-squares estimator for a system of equations with singular values $\left\{w_{k}, k=1 \ldots n\right\}$ is given by (Press et al. 1992)

$\sigma_{\text {total }}^{2}=\sigma^{2} \sum_{k=1}^{n} \frac{1}{w_{k}^{2}}$

where $\sigma^{2}$ is the variance of the individual data values. If we arrange the singular values in descending order, then the contribution to the variance of the degenerate "direction" of the error ellipse in the model space is given by $\sigma^{2} / w_{n}^{2}$ whereas the mean contribution of the remaining directions is

$\frac{\sigma^{2}}{n-1} \sum_{k=1}^{n-1} \frac{1}{w_{k}^{2}}$.

We therefore define the "degeneracy factor" $\kappa$ as

$\kappa=\frac{\frac{1}{w_{n}^{2}}}{\frac{1}{n-1} \sum_{k=1}^{n-1} \frac{1}{w_{k}^{2}}}$.

Just like the condition number, a degeneracy factor of unity corresponds to a completely non-degenerate solution while an infinite degeneracy factor corresponds to a completely degenerate solution. Unlike the condition number, intermediate degeneracy factors can be used to understand when the degeneracy is at an acceptable level, as will be shown in Sect. 5 .

Figure 3 shows how $k$ varies when the declination range of the targets observed is changed. Declination ranges with different upper limits $\delta_{\max }$ are shown; the case $\delta_{\max }=90$ is perhaps of most interest when studying a polar or mid-latitude interferometer sited in the Northern hemisphere (clearly these results can be used for Southern hemisphere interferometers by exchanging $\delta$ for $-\delta$ everywhere). The values of $\kappa$ plotted are for simulated observations of $N_{\text {star }}=20$ stars; when the value of $N_{\text {star }}$ was varied between 10 and 80 but the declination range of the stars was kept the same, the values of $\kappa$ were found to remain approximately constant.

Figure 3 confirms that, as expected from the earlier heuristic analysis, the degeneracy factor is large when the star declinations are restricted to a range near to the pole, and $\kappa$ generally decreases when a larger range of declinations is covered. The heuristic exploration indicated that a degeneracy should be present when the stars observed are all much less than a radian from the pole, but it can be seen that $\kappa$ remains significantly greater than unity even for declination ranges which span from the pole to the equator.

The solution errors corresponding to this degeneracy are given by the "null eigenvector" which is plotted in Fig. 4. It should be remembered that this eigenvector is a unit vector in a space whose axes are declination offsets for different sources, and it represents a set of declination offsets which can be added to any solution and produce relatively little change in the measured data values. Because it is a unit vector, it serves the purpose of representing the ratio of the declination offsets for different stars: the absolute magnitude of these offsets depends on the magnitude of the corresponding singular value. It can be seen from the figure that the offsets are generally smaller for sources near to the pole than for sources near the equator: this can be compared to the $\cot \delta$ form for the degeneracy found in the heuristic analysis.

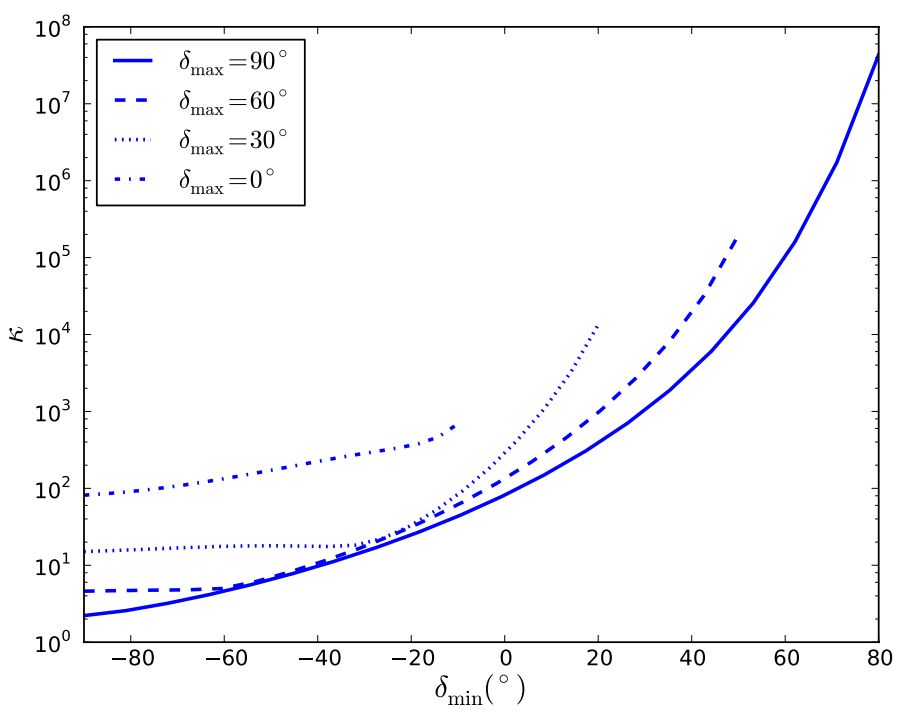

Fig. 3. The "degeneracy factor" $\kappa$ of the astrometric solution for simulated experiments observing stars over the full $360^{\circ}$ hour angle range. In all cases 20 stars, equispaced across a given declination range, were observed. Different lines correspond to different upper limits $\delta_{\max }$ to the declination range as indicated in the legend, and the $x$-coordinate corresponds to the lower limit $\delta_{\min }$ of the range used. In all simulations a single baseline with equal polar and equatorial components was used.

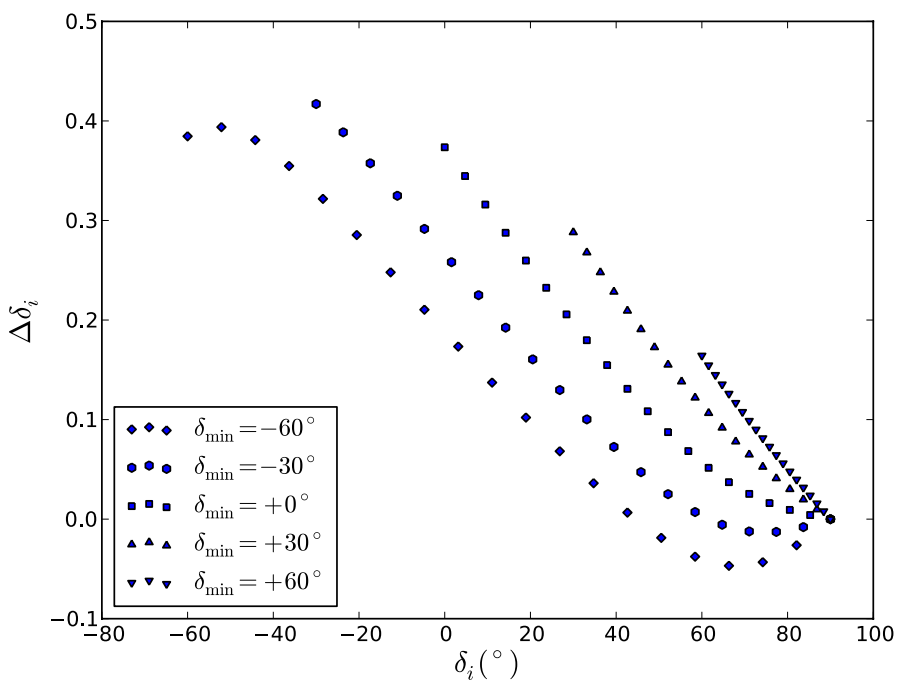

Fig. 4. "Null eigenvectors" corresponding to the smallest singular value for a given astrometric experiment. Each eigenvector consists of a set of declination offsets $\Delta \delta_{i}$ for each of the set of 20 stars in the experiment. These offsets are plotted as a function of the star declination $\delta_{i}$. For simplicity the corresponding baseline offset, which is also part of the eigenvector, is not shown. The different eigenvectors shown correspond to experiments with different minimum observed star declinations $\delta_{\min }$; the maximum declination observed is $\delta_{\max }=90^{\circ}$ in all cases.

\subsection{The effect of hour-angle coverage}

The above results were derived assuming that all the stars can be observed for a full diurnal cycle. While this scenario is directly applicable to interferometers situated in polar regions or on rotating space platforms, it is a less good approximation to groundbased observations at mid latitudes which form the bulk of existing interferometric observations. In these cases, the hour-angle coverage for a given target is restricted by the Earth's horizon, and as an object approaches the horizon refraction errors caused 
by the Earth's atmosphere make observations increasingly unreliable (MacMillan \& Ma 1997).

A restriction in the range of hour angles over which a target can be observed with any given baseline will limit the range of values of $H_{\mathrm{ijk}}$, the interferometer hour angle (IHA) over which the delay is sampled. However in the majority of practical observations, a given target is observed on more than one baseline and, if these baselines have different hour angles with respect to the meridian, then it may still possible observe a given object over the full 360-degree range in IHA. Thus one might then suppose that the previous analysis would provide a good approximation to realistic observation scenarios.

To determine if the declination degeneracy identified previously is still present under more realistic conditions, simulations were performed of zenith-distance-limited observations at mid latitudes. The simulated interferometer was sited at a latitude of $+30^{\circ}$, and was assumed to be small compared with the radius of the Earth so that any given star had the same elevation when seen from any antenna (unlike a VLBI interferometer where the elevation of a given source at any given instant varies from antenna to antenna).

The simulated interferometer consisted of two independent baselines, one running due North-South one running due East-West. The North-South baseline had unit polar component an equatorial component of $1 / \sqrt{3}$, consistent with a "groundhugging" interferometer at $30^{\circ}$ latitude, while the East-West baseline had no polar component and unit equatorial component. It should be noted that in this arrangement the equatorial components of the two baselines are orthogonal, thereby diversifying the range of IHA values observed.

Observations of a set of 20 stars spaced uniformly in declination were simulated. For each star, delay measurements were made uniformly over the hour angle range accessible for that star within the given zenith-distance limit. The full set of equations represented by Eq. (3), as opposed to the restricted set of equations represented by Eqs. (12) and (13) used earlier, was solved using SVD and the singular values and the eigenvectors were examined in each case.

It was found that the behaviour seen with restricted zenith distance observations was similar to that seen for full hour-angle coverage. Figure 5 shows the spectrum of singular values for observations when the observations are restricted to targets within $75^{\circ}$ of the zenith. It can be seen there is a partial degeneracy similar to that seen with no zenith-distance restrictions, indicated by a singular value significantly smaller in magnitude than the majority of the other singular values (with the exception of the zero singular value corresponding to the well-known degeneracy in right ascension, which was ignored in this analysis). Simulations with different zenith angle limits and declination ranges exhibited similar qualitiative behaviour.

The degenerate singular value in all cases corresponded to a "null eigenvector" which was in the form of a declination distortion and corresponding baseline distortion; the right ascension component of this eigenvector was zero to machine precision. The degeneracy factors and the shape of the null eigenvectors for zenith-distance-limited observations were found to be close to those seen for observations with the same declination limits but full diurnal coverage, indicating that it is the declination limit that determines the form of the degeneracy more than the hour-angle limit.

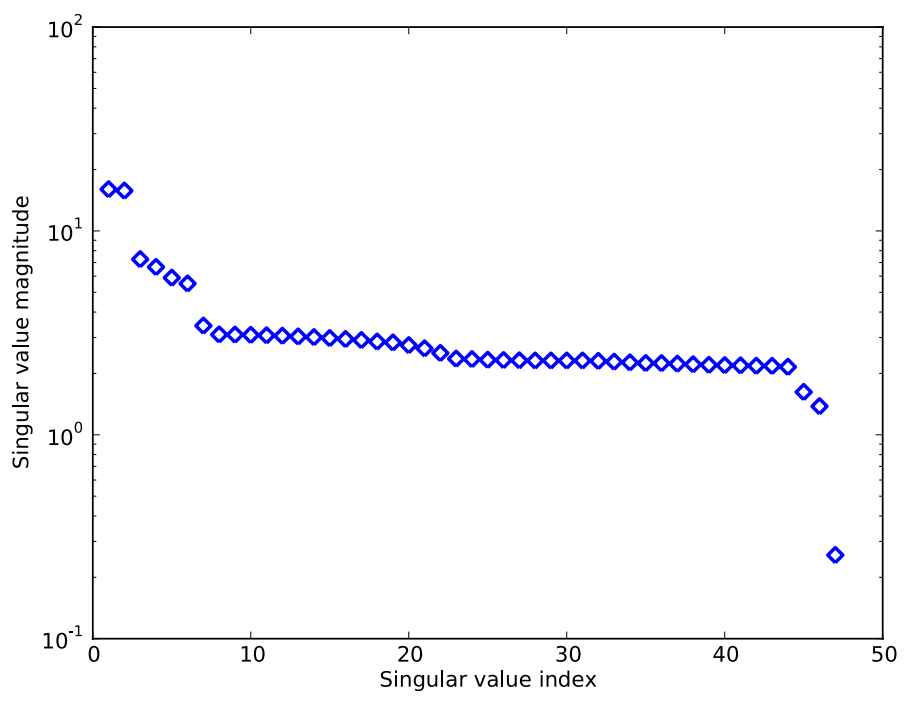

Fig. 5. Singular values for a simulated interferometric experiment where the observations were limited in hour angle so that targets were always observed when they were within $75^{\circ}$ of the zenith. The singular values are plotted in decreasing order. The interferometer had North-South and East-West baselines as explained in the text. The 20 targets were uniformly spaced in the declination range $0^{\circ}$ to $90^{\circ}$ and were observed at 8 uniformly-spaced hour angles within the available HA range. The singular value corresponding to the degeneracy in the zero point of RA is not shown, so a total of 47 singular values are plotted (the degrees of freedom of the solution consist of right ascenscions and declinations for each of the 20 targets and 4 baseline parameters for each of 2 baselines).

\section{Implications for interferometric astrometry}

The analysis above has shown that an idealised geometric model for interferometric astrometry predicts a significant partial degeneracy in declination for these measurements even when the range of source declinations extend from the pole to the equator. The simulations of more realistic observations show that this ideal model acts as a good predictor of the level of degeneracy even when less-than-ideal hour-angle coverage is present. The following subsections consider the consequences of the existence and form of the degeneracy in a number of practical applications.

\subsection{The design of astrometric experiments}

Figure 3 shows that the ideal interferometric experiment should observe sources from pole-to-pole in order to minimise the the magnitude of the declination degeneracy. This is not practical in many cases and so an alternative is to derive an estimate for the minimum declination coverage which gives an acceptable level for the degeneracy.

A given level of degeneracy may be considered acceptable if the degeneracy contributes less than or the same amount to the total variance in the solution as all the other error sources combined. The degeneracy factor $\kappa$ is a measure of the ratio of the variance contributed by the degenerate component of the model to that contributed by an average non-degenerate model component, so $\kappa$ needs to be less than or of the order of the number of other parameters which contribute to the declination errors for the above condition to be achieved.

It would seem reasonable to assume that the non-degenerate parameter errors are roughly equally distributed between errors in declination and errors in RA and it is often the case the number of baselines is small compared to the number of sources. 
Under these conditions the acceptable level for the degeneracy parameter will be approximately equal to the number of sources. Thus, for example, if an experiment observes 20 sources then the level of degeneracy may be considered acceptable if $\kappa \lesssim 20$; from Fig. 3 we can see that this can be achieved by observing sources equally spaced between declinations of $-20^{\circ}$ and $+90^{\circ}$.

Combining observations from a Southern-hemisphere interferometer with those from a Northern-hemisphere interferometer may alleviate the problem of the poor declination coverage of either interferometer alone to some extent. However, it is not clear how well this would work, since the sources in common to the two interferometers are those near the equator; it can be seen from Fig. 4 that sources near the equator will suffer from the worst errors in both interferometers.

When the conditions for adequate declination coverage are not met, the effects can be dramatic. Hummel et al. (1994) made optical interferometric observations with an intrinsic accuracy level estimated to be at the 0.01 arcsec level, but because of limitations in the declination coverage, the declination degeneracy introduced errors at the level of several arcseconds. This experiment provided the initial motivation for this study.

\subsection{Antarctic and East-West interferometers}

For an East-West interferometer the values of $p_{j}$ are all zero and so the family of equations given by Eq. (13) provide no additional constraints on the degeneracy proportional to $\cot \delta$ given in Eq. (15). As a consequence, large distortions in declination near the equator are possible independent of the declination range observed. Ryle \& Elsmore (1973) noted that measurement of the declinations of sources near the equator with an East-West interferometer was prone to error, but turned the argument on its head: by measuring the interferometric delays of sources within 30 arcmin of the equator whose declinations were known with moderate precision they were able to accurately determine the equatorial component of their baseline.

The same result applies to the interferometers that are being proposed for siting in Antarctica (Lloyd et al. 2002) since for any ground-hugging interferometer the polar component of the baseline will be very small in comparison to the equatorial component. In the case of Antarctic interferometers the solution adopted by Ryle and Elsmore is less practical: equatorial sources will be near the horizon where errors due to atmospheric refraction are likely to be large. An alternative way to break this degeneracy would be to use astrometric measurements of higherdeclination sources made using other methods with comparable accuracy, for example space missions.

\subsection{Differential astrometry}

The highest-precision astrometric measurements often come from differential astrometry (Shao \& Colavita 1992), where the relative positions of two sources which are separated by relatively narrow angles are measured. These measurements are less susceptible to a number of sources of systematic error and this is also true in the case of the declination degeneracy: it can be seen from Fig. 4 that the declination errors of sources of similar in declination are similar, so the differential declination error will be small compared to the global declination error caused by this degeneracy.

Nevertheless the declination degeneracy may still cause problems in experiments where a global astrometric solution is used to determine the baseline used for the differential measurement. Since the degeneracy causes a distortion both of the measured declinations and of the baselines, then a severe degeneracy caused by too narrow a declination coverage and/or a lack of sufficient polar component of the baselines could be sufficient to affect the precision of differential measurements. Therefore caution still needs to be exercised when choosing the set of sources used to measure the interferometer baseline.

\subsection{Comparing catalogues}

To cope with the well-known degeneracy in right ascension, it is conventional to solve the interferometric equations for a set of model variables which can be constrained by the data (the "range space"), namely the RAs of the stars relative to one another and the equatorial rotations of the baselines relative to one another, and in addition to specify a set of model variables for which the data are unable to provide any constraints (the "null space"), namely the average RA offsets of the stars and the average rotational offsets of all the baselines.

When comparing catalogues of stars from two different experiments it is natural therefore to allow for the unconstrained degree of freedom in both catalogues by rotating the catalogues relative to one another in RA to achieve the best agreement between them. When comparing two catalogues where one or both are subject to the declination degeneracy, a similar procedure could be adopted, since the declination degeneracy, while only partial, may comprise a significant "systematic error" (in the sense of an error which is strongly correlated between stars) for a given catalogue. In this case however, the catalogues cannot simply be rotated relative to one another since the under-constrained "direction" in parameter space does not correspond to a uniform motion of all the stars in a given catalogue. Instead, motion in the along the "null vector" consists of a small declination shift near the poles and a much larger shift near the equator as shown in Fig. 4.

When comparing two interferometric catalogues one needs to "move" the declination solutions along their respective "null directions" until the catalogues agree as well as possible. This motion is achieved by changing the scale factor by which the declination eigenvector is magnified before being added as an offset to the catalogue positions. It should be re-emphasized that this is an equivalent procedure to shifting catalogues in RA - it involves adjusting a single degree of freedom to make a much larger number of parameters fit. The main difference between the RA and declination degeneracies lies mainly in our intuitive ability to grasp what transformations are being applied to the solutions, not a difference in the order of the problem.

A second difference is that the null eigenvectors for interferometric catalogues are currently not published. In some cases, this may be because for the very large datasets comprising, for instance, the ICRF, the magnitude of the declination degeneracy is sufficiently small to be neglected.

However there is some evidence suggesting that an effect similar to the declination degeneracy described here may be important. Studies comparing radio source catalogues have found that ad-hoc methods which systematically shift source declinations by different amounts depending on declination are effective in improving the agreement between different catalogues (Arias \& Bouquillon 2004; Sokolova \& Malkin 2007), and it is possible this is partly an effect of a declination degeneracy.

In addition, radio-VLBI experiments often show systematic errors in declination for sources near the equator. These can be removed by modelling these as additional refraction corrections near the equator (MacMillan \& Ma 1997), but could perhaps 
include a component arising from a partial degeneracy in declination.

\subsection{Geodesy and Earth orientation}

Interferometric astrometry yields measurements of the relative coordinates of Earth-based telescopes as well as the positions of celestial sources, and this can be used as part of geodetic programmes which aim to measure motions of the Earth's crust and the orientation of the Earth with respect to an "inertial" frame. The declination degeneracy consists of distortions to baselines as well as source positions, and so this degeneracy has the potential to affect both astrometric and geodetic measurements.

While the degeneracy in RA leads to a global rotation of the baseline coordinates which can be straightforwardly dealt with, the declination degeneracy can lead to an apparent change in the lengths of the baselines in a correlated way, which could be misinterpreted in terms of global changes of shape of the Earth. Thus the same caution about choosing an acceptable declination range for the celestial sources applies to both astrometric and geodetic experiments.

An important application of interferometry is to determine Earth orientation, and in particular the motion of the Earth's rotation axis (Arias et al. 1995). The shape of the degenerate eigenvectors shown in Fig. 4 gives rise to a useful property for the measurement of polar motion. Although absolute source declinations are badly determined at low declinations, at declinations near the pole, the error becomes smaller. Thus measuring polar motion through preferentially monitoring the declination motion of sources close to the pole can reduce the effects of the declination degeneracy to arbitrarily small values. In this case it may be possible to determine polar motion to a high degree of accuracy even when the declination coverage is poor.

\section{Conclusions}

It has been shown that interferometric astrometry experiments are subject to a degeneracy which exists under a wide range of experimental conditions. This degeneracy results in a correlated set of distortions in the measured declinations of the celestial targets, together with a corresponding distortion of the measured baselines. These distortions have the potential to affect the design of interferometric astrometric experiments and the interpretation of their results, and the implications of this have been discussed in some example cases.

The results given here are from a limited number of geometries and observing scenarios, so can only be used as a guide as to whether the degeneracy might be important in any particular case. It is recommended that astrometric experiments are investigated on a case-by-case basis to understand the importance of this degeneracy and how to ameliorate its effects. This study has shown that SVD is a powerful tool for such investigations.

Acknowledgements. I would like to thank C. Hummel for the discussions which originally motivated this study, G. Kaplan for comments on an early draft, C. Haniff for careful reading of the manuscript and F. Adria for his contributions to the heuristic exploration of the range and null spaces of partially-degenerate problems. I would also like to thank the anonymous referee for pointing out the question as to whether telescope-based formalisms and baseline-based formalisms are subject to the same degeneracies.

\section{Appendix A: Telescope-based formalisms}

The formalism used in Eq. (2) can be expressed in vector form as

$d_{i j k}=\hat{\boldsymbol{s}_{i}} \cdot \boldsymbol{D}_{j k}+c_{j}+n_{i j k}$

where $\hat{\boldsymbol{s}}_{i}$ is the direction of $\operatorname{star} i, \boldsymbol{D}_{j k}$ is the vector representing baseline $j$ at time $k, c_{j}$ is a baseline-dependent offset and $n_{i j k}$ is a noise value for the given measurement. Alternatively, we can write Eq. (A.1) in the form

$d_{i k}^{l m}=\hat{\boldsymbol{s}}_{i} \cdot\left(\boldsymbol{X}_{k}^{l}-\boldsymbol{X}_{k}^{m}\right)+c^{l}-c^{m}+n_{i k}^{l m}$

where $l$ and $m$ denote the pair of telescopes which form baseline $j$, and where the telescopes have coordinates $\boldsymbol{X}_{k}^{l}$ and $\boldsymbol{X}_{k}^{m}$ respectively at time $k$ and have associated clock offsets $c^{l}$ and $c^{m}$ respectively. The absolute positions of the telescopes cannot be determined from the data, but this can be dealt with by setting the coordinates of one or more of the telescopes to some fixed value.

The baseline-based formalism of Eq. (A.1) and the telescope-based formalism of Eq. (A.2) give identical results where each telescope is only ever used to form fringes with one other telescope, but the results may differ when this is not true. This is because the information that one telescope position is involved in more than one baseline is naturally included in the telescope-based equations but requires extra constraints to be added to the baseline-based equations. However, the following argument indicates that if a degeneracy exists in the baselinebased equations without applying these additional constraints, then a similar degeneracy always exists in the telescope-based equations.

The existence of a degeneracy in the baseline-based equations means that there exists a set of non-zero offsets $\left\{\Delta \boldsymbol{s}_{i}\right\}$, $\left\{\Delta \boldsymbol{X}_{k}^{l}-\Delta \boldsymbol{X}_{k}^{m}\right\}$ and $\left\{\Delta c^{l}-\Delta c^{m}\right\}$ such that

$$
\begin{aligned}
& \left(\hat{\boldsymbol{s}}_{i}+\Delta \boldsymbol{s}_{i}\right) \cdot\left(\boldsymbol{X}_{k}^{l}+\Delta \boldsymbol{X}_{k}^{l}-\boldsymbol{X}_{k}^{m}-\Delta \boldsymbol{X}_{k}^{m}\right)+c^{l}+\Delta c^{l}-c^{m}-\Delta c^{m}= \\
& \hat{\boldsymbol{s}_{i}} \cdot\left(\boldsymbol{X}_{k}^{l}-\boldsymbol{X}_{k}^{m}\right)+c^{l}-c^{m}+\eta_{i k}^{l m}
\end{aligned}
$$

for a particular set of a priori star positions and baseline parameters $\left\{\hat{\boldsymbol{s}}_{i}\right\},\left\{\boldsymbol{X}_{k}^{l}-\boldsymbol{X}_{k}^{m}\right\}$ and $\left\{c^{l}-c^{m}\right\}$, and where $\eta_{i k}^{l m}$ is an offset which is zero for a full degeneracy and small for a partial degeneracy. For simplicity the case of $\eta_{i k}^{l m}=0$ will be treated initially, and an indication of how the argument can be generalised for non-zero $\eta_{i k}^{l m}$ will be given later.

Ignoring second-order terms, Eq. (A.3) can be written

$\Delta \boldsymbol{s}_{i} \cdot\left(\boldsymbol{X}_{k}^{l}-\boldsymbol{X}_{k}^{m}\right)+\hat{\boldsymbol{s}_{i}} \cdot\left(\Delta \boldsymbol{X}_{k}^{l}-\Delta \boldsymbol{X}_{k}^{m}\right)+\Delta c^{l}-\Delta c^{m}=0$

in the case of a full degeneracy. The simplest arrangement which illustrates the difference between the telescope-based and baseline-based formalisms is one where there are three telescopes and where delay measurements are made on all three baselines between these telescopes. In this case, the information present in the baseline-based formalism can be represented by modelling three independent pairs of telescopes with coordinates $\left\{\boldsymbol{X}_{k}^{1}, \boldsymbol{X}_{k}^{2}, \boldsymbol{X}_{k}^{3}, \boldsymbol{X}_{k}^{4}, \boldsymbol{X}_{k}^{5}, \boldsymbol{X}_{k}^{6}\right\}$. Because the data actually come from 3 rather than 6 telescopes, the a priori baselines will form a closed triangle so that

$\left(\boldsymbol{X}_{k}^{1}-\boldsymbol{X}_{k}^{2}\right)+\left(\boldsymbol{X}_{k}^{3}-\boldsymbol{X}_{k}^{4}\right)+\left(\boldsymbol{X}_{k}^{5}-\boldsymbol{X}_{k}^{6}\right)=0$.

The telescope-based formalism adds the additional information that the telescope coordinates are not independent from one another so that, for example, $\Delta \boldsymbol{X}_{k}^{3}=\Delta \boldsymbol{X}_{k}^{2}$ and so on. As a result, any degeneracy must also satisfy

$\left(\Delta \boldsymbol{X}_{k}^{1}-\Delta \boldsymbol{X}_{k}^{2}\right)+\left(\Delta \boldsymbol{X}_{k}^{3}-\Delta \boldsymbol{X}_{k}^{4}\right)+\left(\Delta \boldsymbol{X}_{k}^{5}-\Delta \boldsymbol{X}_{k}^{6}\right)=0$ 
and

$\left(\Delta c_{k}^{1}-\Delta c_{k}^{2}\right)+\left(\Delta c_{k}^{3}-\Delta c_{k}^{4}\right)+\left(\Delta c_{k}^{5}-\Delta c_{k}^{6}\right)=0$.

Equations (A.6) and (A.7) are additional constraints on the degeneracy which are not explicitly present within the baselinebased formalism and so it might at first sight appear possible that a degeneracy which is seen in the baseline-based formalism may not be compatible with the all the constraints present in the telescope-based formalism. However, Eqs. (A.4) and (A.5) can be combined to show that

$$
\begin{aligned}
\hat{s_{i}} \cdot\left(\Delta \boldsymbol{X}_{k}^{1}-\Delta \boldsymbol{X}_{k}^{2}+\Delta \boldsymbol{X}_{k}^{3}-\Delta \boldsymbol{X}_{k}^{4}+\Delta \boldsymbol{X}_{k}^{5}-\Delta \boldsymbol{X}_{k}^{6}\right) & \\
& +\Delta c_{k}^{1}-\Delta c_{k}^{2}+\Delta c_{k}^{3}-\Delta c_{k}^{4}+\Delta c_{k}^{5}-\Delta c_{k}^{6}=0 .
\end{aligned}
$$

Since Eq. (A.8) is true for multiple different values of $\hat{\boldsymbol{s}}_{i}$, it can only be satisfied if Eqs. (A.6) and (A.7) are both individually satisfied. Thus the additional constraints present in the telescope-based formalism are satisfied by any full degeneracy in the baseline-based formalism, so any degeneracy in the latter formalism must also exist in the former formalism.

For the case of a partial degeneracy where $\eta_{i k}^{l m}$ is small but non-zero, the above reasoning can be modified to show that the elongated "direction" of the error ellipsoid in solution space corresponding to the partial degeneracy lies almost parallel to the "hyperplanes" of constraints given by Eqs. (A.6) and (A.7). The error ellipsoid for the telescope-based equations will lie on the intersection of the error ellipsoid for the baseline-based equations and these hyperplanes, and so there will be a degeneracy of similar magnitude and direction in both the the telescope-based and baseline-based formalisms.
For arrays of more than 3 telescopes, the additional constraints present in the telescope-based formalism can be represented in the form of the set of "closure constraints" such as those given in Eqs. (A.7) and (A.8) for all the triplets of telescopes in the array, since the constraints from any higher-order closure geometries such as quadrilaterals will be linearly dependent on the constraints from the triplets which can be made from the same telescopes. For each of these triplets, the results given above show that any baseline-based degeneracy satisfies (or nearly satisfies, in the case of a partial degeneracy) the closure constraints, and hence that the degeneracy is also a degeneracy of the telescope-based formalism. Thus the analysis given in this paper is a good guide to the degeneracies present whichever formalism is used.

\section{References}

Arias, E. F., \& Bouquillon, S. 2004, A\&A, 422, 1105

Arias, E. F., Charlot, P., Feissel, M., \& Lestrade, J.-F. 1995, A\&A, 303, 604

Golub, G. H., \& Reinsch, C. 1970, Num. Math., 14, 403

Hummel, C. A., Mozurkewich, D., Elias, N. M., et al. 1994, AJ, 108, 326

Launhardt, R., Henning, T., Queloz, D., et al. 2007, Proc. International Astronomical Union, 3, 417

Lloyd, J. P., Oppenheimer, B. R., \& Graham, J. R. 2002, PASA, 19, 158

Ma, C., Arias, E. F., Eubanks, T. M., et al. 1998, AJ, 116, 516

MacMillan, D. S., \& Ma, C. 1997, Geophys. Res. Lett., 24, 453

Pearson, T. 1991, Introduction to the Caltech VLBI Programs

Press, W. H., Teukolsky, S. A., Vetterling, W. T., \& Flannery, B. P. 1992, Numerical recipes in FORTRAN, The art of scientific computing (Cambridge: Cambridge University Press)

Ryle, M., \& Elsmore, B. 1973, MNRAS, 164, 223

Shao, M., \& Colavita, M. M. 1992, A\&A, 262, 353

Sokolova, J., \& Malkin, Z. 2007, A\&A, 474, 665

Sovers, O. J., Fanselow, J. L., \& Jacobs, C. S. 1998, Rev. Mod. Phys., 70, 1393

Thompson, A. R., Moran, J. M., \& Swenson, Jr., G. W. 2001, Interferometry and Synthesis in Radio Astronomy, 2nd edition (New York: Wiley) 